\title{
Kinetics of fragmentation
}

\author{
Z Cheng and S Redner \\ Center for Polymer Studies and Department of Physics, Boston University, Boston, MA \\ 02215 , USA
}

Received 17 August 1989

\begin{abstract}
A general discussion of the kinetics of continuous, irreversible fragmentation processes is presented. For a linear process, where particle breakup is driven by an external force, we develop a scaling theory to describe the evolution of the cluster size distribution. We treat the general case where the breakup rate of a cluster of mass $x$ varies as $x^{\lambda}$. When $\lambda>0$, corresponding to larger clusters more likely to break up, the scaled cluster size distribution, $\phi(x)$, decays with the scaled mass, $x$, as $x^{-2} \exp \left(-a x^{i}\right)$, as $x \rightarrow \infty$. For small mass, $\phi(x)$ has the $\log$-normal form, $\exp \left(-a \ln ^{2} x\right)$, if the breakup kernel has a small-size cutoff, while $\phi(x)$ has a power-law tail in the absence of a cutoff. We also show that a conventional scaling picture applies only for the case $\lambda>0$. For $\lambda<0$, we develop an alternative formulation for the cluster size distribution, in which the typical mass scale is determined by the initial condition. In this regime, we also investigate the nature of a 'shattering' transition, where mass is lost to a 'dust' phase of zero-mass particles. We also study the kinetics of a nonlinear, collision-induced fragmentation process. We analyse the asymptotic behaviours of a simple-minded class of models in which a two-particle collision results in either: (1) both particles splitting into two equal pieces, (2) only the larger particle splitting in two, or (3) only the smaller particle splitting. We map out the kinetics of these models by scaling arguments and by analytic and numerical solutions of the rate equations. Scaling is found to hold for different ranges of homogeneity index for the three models.
\end{abstract}

\section{Introduction and summary}

Fragmentation is a continuous and irreversible kinetic process which occurs in many physical situations such as polymer degradation [1-4], liquid droplet breakup [5], crushing or grinding of rocks $[6,7]$ and combustion [8]. A basic goal of this paper is to provide a general theoretical description of the evolution of the distribution of fragment sizes that results from such breakup processes. A basic theoretical approach to describe this process is by the rate equations. These are an approximation of a meanfield character, as fluctuations are ignored. Fragments are assumed to be distributed homogeneously at all times throughout the system, i.e. there is perfect mixing, and the variability of cluster shapes is ignored. Thus the mass is the only dynamical variable that characterises a given fragment in the rate equation approach.

The rate equations have been extensively studied in the mathematics, physics and applied science literatures. By a mathematical approach, Filippov [9] found general conditions for which the fragment size distribution approaches a limiting form at long times. Ziff and McGrady [10-12] have recently applied a statistical mechanical viewpoint to fragmentation and have obtained new insights which parallel recent progress made in the investigation of aggregation phenomena. A variety of basic 
results have also been obtained for experimentally-and theoretically-motivated models $[10-12,17,18]$. An important feature of fragmentation is that the typical cluster size decreases with size due to breakup. The vanishing of the typical size is analogous to the vanishing of the inverse correlation length for a system near a second-order phase transition. Thus at long times, one might anticipate that scaling and universality can be invoked to describe the nature of the cluster size distribution.

Our treatment is based on applying scaling to obtain asymptotic information about the solutions to the rate equations for a general class of models. We will argue that only a few details of the breakup process are relevant in determining basic features of the fragment size distribution at long times. Thus the scaling approach naturally leads to a universal way of describing fragmenting systems. Our approach parallels analogous developments in the investigation of the rate equations for the kinetics of the inverse process of aggregation [18-21]. We will give a more detailed account of our earlier work [17] and present a number of new results.

One basic classifying feature of fragmentation is whether breakup is driven only by a homogeneous external agent, or whether additional influences also play a role. The former leads to an inherently linear process, while the additional driving influences that one might envision in fragmentation are often nonlinear in character. One example is fragmentation induced by collisions between fragments. This might arise in an explosive-type process, or perhaps in the breakup of small eddies in a turbulent fluid flow [22]. As an idealisation of such a situation, we consider collision-induced fragmentation, in which fragmentation products continuously participate in repeated collisions and fragmentations $t$. While a purely collision-induced process may be too idealised to be of direct practical relevance, the phenomenology of such processes is quite rich. Furthermore, there are parallels with the rate equations of the inverse process of aggregation, since both processes are driven by a bimolecular rate. Thus studies of collision-induced fragmentation may provide a useful first step in understanding nonlinear effects in fragmentation kinetics.

The outline of this paper is as follows. In section 2 , we define the linear and the nonlinear, collision-induced fragmentation models, and write their corresponding rate equations. For linear fragmentation, we consider homogeneous systems in which a cluster of mass $x$ breaks up at a rate which varies as $x^{i}$, and in which the rate of production of a fragment of mass $x$, from the breakup of a cluster of mass $y$, depends only on the ratio $x / y$. For collision-induced fragmentation, we focus on a particular class of 'splitting' models in which a two-particle collision results in either: (1) both particles splitting exactly in two, (2) only the larger particle splitting, or (3) only the smaller particle splitting. These models retain the essential nonlinearity and generality of collision-induced fragmentation, while being simple enough to solve.

In section 3, we discuss basic features of scaling solutions, including the singular cases where the inverse moments of the cluster size distribution fail to converge. In section 4 , we derive scaling solutions to the rate equations for linear fragmentation for systems with positive homogeneity index, $\lambda>0$. The asymptotic form of the particle-size distribution at large size is almost completely determined by the value of $\lambda$. In the small-size limit, we determine the general conditions on the relative breakup rate, which delineates between the classical log-normal form or a power-law form for the small mass tail of the distribution. The former is characteristic of a random multiplicative process, and this has been invoked to describe many rock crushing

† See, for example, [23] for a particular limit of nonlinear fragmentation. 
processes (see [24], also [25] and references therein). In section 5, we discuss general properties of the solutions to the rate equations for linear fragmentation when $\lambda<0$. The basic new feature of this case is that the typical size is now determined by the initial mass distribution rather than evolving dynamically. We also discuss the criterion for the existence of a 'shattering' transition, [9,12] in which mass is lost to a phase of zero-size particles. In section 6 , we derive the asymptotic solutions for the collisioninduced splitting models. We also investigate the conditions for which the fragment size distribution can be described by a scaling form. In section 7 , we test our theoretical predictions by direct numerical integration of the rate equations. Finally, in section 8 , we give our basic conclusions and offer a few suggestions for additional work.

\section{Rate equations for linear and collision-induced fragmentation}

\subsection{Linear fragmentation}

Denote by $c(x, t)$ the concentration of clusters of mass $x$ at time $t$. When particle breakup takes place independently and homogeneously as a result of an external source, the evolution of $c(x, t)$ is described by the linear integrodifferential equation [9-17]

$$
\frac{\partial c(x, t)}{\partial t}=-a(x) c(x, t)+\int_{x}^{x} c(y, t) a(y) f(x \mid y) d y .
$$

Here $a(x)$ is the overall rate at which $x$ breaks, i.e. $a(x) \mathrm{d} t$ is proportional to the probability that an $x$-mer breaks in a time interval $\mathrm{d} t$, while $f(x \mid y)$ is the relative breakup rate, i.e. the conditional probability at which $x$ is produced from the breakup of $y$. The first term on the right-hand side of equation (1) therefore accounts for the loss of $x$-mers due to their breakup, while the second term accounts for the gain of $x$-mers by the breakup of particles with mass larger than $x$. We will typically consider initial mass distributions which are bounded; this initial mass turns out to be relevant in the asymptotic form of the cluster size distribution only when $\lambda<0$.

The cluster size distribution that results from equation (1) is determined by the details of the kernels $a(x)$ and $f(x \mid y)$. With their form left unspecified, it is difficult to make general statements about the solutions to the rate equations (see, however, [9]). Therefore we restrict ourselves to homogeneous kernels henceforth. Homogeneity implies that the overall breakup rate depends on the mass of a fragment as $a(x)=x^{\lambda}$, where $\lambda$ is the homogeneity index. Homogeneity also implies that $f(x \mid y)$ depends only on the ratio of the mass of the product to the mass of the initial cluster, i.e. $f(x, y) \propto y^{-1} b(x / y)$. Notice that the integral $\int_{0}^{\infty} b(x) \mathrm{d} x$ equals the average number of fragments produced in a single breakup event. This quantity can be either finite or infinite in our results, although only the former case is physical. Mass conservation also imposes the condition $\int_{0}^{1} x b(x) \mathrm{d} x=1$.

A basic reason for restricting ourselves to homogeneous kernels is that this choice includes most physical situations. More importantly, scaling provides a simple and powerful tool for analysing the asymptotic behaviour for homogeneous fragmenting systems. 


\subsection{Collision-induced fragmentation}

For breakup driven by collisions between fragments, the interaction details between two clusters are now specified by a collision kernel $K(x, y)$, which gives the rate at which an $x$-mer and a $y$-mer meet, and a breakup kernel $B(x \mid y, z)$, which specifies the rate at which $x$-mers are generated as result of the breakup of a $y$-mer in a collision between a $y$-mer and a $z$-mer. The rate equations for $c(x, t)$ now are [17]

$$
\frac{\partial c(x, t)}{\partial t}=-c(x, t) \int_{0}^{\infty} K(x, y) c(y, t) \mathrm{d} y+\int_{0}^{\infty} \mathrm{d} z \int_{x}^{\infty} K(y, z) B(x \mid y, z) c(y, t) c(z, t) \mathrm{d} y .
$$

The first term on the right-hand side accounts for the loss of $x$-mers because of collisions between $x$-mers with the remaining particles in the system, while the second term describes the gain of $x$-mers due to collisions in which the mass of the particle undergoing breaking is larger than $x$. In this nonlinear model, the collision kernel is symmetric, i.e. $K(x, y)=K(y, x)$, while mass conservation implies that $y=\int_{0}^{y} x B(x \mid y, z) \mathrm{d} y$.

In order to determine the possible asymptotic behaviours of this model, it is necessary to specify the functional forms of the collision and breakup kernels. For this purpose, we appeal to the analogy with aggregation processes and consider homogeneous systems because of their generality and because considerable information can be deduced once the scaling behaviour of the kernels as a function of their arguments is given. Accordingly, we consider collision kernels which can be written as

$$
K(a x, a y)=a^{j} K(x, y) \text {. }
$$

For the breakup kernel, many forms for $B(x \mid y, z)$ correspond to physically reasonable microscopic processes. Note, however, that the breakup kernel must satisfy

$$
B(a x \mid a y, a z)=a^{v} B(x \mid y, z)
$$

where $v=-1$ is required by mass conservation. Equation (2) can be reduced to the rate equations of a linear fragmentation process for particular classes of kernels, such as those in which $B(x \mid y, z)$ has no dependence on $z$, and in which $K(x, y)$ has the product form $K(x, y)=f(x) f(y)$. Under these restrictions, any linear fragmentation problem with a homogeneous breakup kernel can be obtained by a suitable choice of $B(x \mid y, z)$.

For the purposes of detailed study, we concentrate on a class of 'splitting' models which exhibit the essential nonlinearity of a collision-induced fragmentation process, but are simple enough to be analysed. In these models, the particle(s) which undergo breaking always split into two equal halves. There are three generic cases to consider. Model I. Both incident particles split in two as a result of a collision. This situation is described by the kernels

$$
\begin{aligned}
& K(x, y)=x^{\lambda / 2} y^{\lambda / 2} \\
& B(x \mid y, z)=2 \delta(x-y / 2) .
\end{aligned}
$$

Model II. Only the larger of the two incident particles splits in two. In the case where the two particles have the same mass, then only one of the particles splits. The corresponding collision and breakup kernels are

$$
\begin{aligned}
& K(x, y)= \begin{cases}x^{\lambda} & \text { if } x \geq y \\
y^{\lambda} & \text { otherwise. }\end{cases} \\
& B(x \mid y, z)= \begin{cases}2 \delta(x-y / 2) & \text { if } y \geq z \\
\delta(x-y) & \text { otherwise. }\end{cases}
\end{aligned}
$$


Model III. Only the smaller particle splits in two. In case of an ambiguity, then only one of the incident particles splits. The corresponding kernels are

$$
\begin{aligned}
& K(x, y)= \begin{cases}x^{\lambda} & \text { if } x \leq y \\
y^{\lambda} & \text { otherwise. }\end{cases} \\
& B(x \mid y, z)= \begin{cases}2 \delta(x-y / 2) & \text { if } z \geq y \\
\delta(x-y) & \text { otherwise. }\end{cases}
\end{aligned}
$$

Models II and III represent the two extreme and opposite situations in which only the larger, or only the smaller of the two incident clusters is susceptible to breakup.

\section{Basic features of the solutions to the rate equations}

There are two general types of solutions to the rate equations; scaling solutions, in which the typical mass evolves continuously, and solutions for which the typical mass is determined by the initial conditions. The value of $\lambda$ determines which of these two types of solutions will be appropriate. We emphasise scaling solutions for several reasons. First, kernels for which the rate equation solutions can be written in a scaling form include most physically relevant cases. Second, the scaling ansatz reduces a two-variable problem to a single-variable problem, thus simplifying the description of fragmentation kinetics. Third, a scaling solution is universal in that it is independent of initial conditions. Thus scaling provides a general classification scheme for the solutions.

The scaling ansatz for the cluster size distribution can be written as [18-21]

$$
c(x, t)=s^{-2} \phi(x / s(t))
$$

where $s(t)$ is a typical (time-dependent) cluster mass, and the exponent -2 is required by mass conservation. In the case of the splitting models, the cluster masses have the discrete form $x=2^{-n}$, with $n$ a positive integer, for a monomer-only initial condition. The transformation between the discrete and continuous form of the cluster size distribution, $c_{n}(t) \mathrm{d} n=c(x, t)(\mathrm{d} x / \mathrm{d} n) \mathrm{d} n$, then leads to the discrete form of the scaling ansatz

$$
c_{n}(t)=s^{-1} \phi\left(2^{-n} / s\right)
$$

It is often the case that the moments of the cluster size distribution can be obtained more conveniently than the complete distribution. Therefore we define the $\alpha$ th-moment of the cluster-size distribution and of the scaling function, $M_{\alpha}(t)$ and $m_{\alpha}$ respectively, by

$$
M_{\alpha}(t)=\int_{0}^{\infty} x^{\alpha} c(x, t) \mathrm{d} x \quad m_{\alpha}=\int_{0}^{\infty} x^{\alpha} \phi(x) \mathrm{d} x .
$$

These are just the Mellin transforms of $c(x, t)$ and $\phi(x)$ (except for a trivial shift of 1 in the definition of $\alpha$ ). When the fragmentation process favours the production of very small fragments, $M_{x}(t)$ may fail to converge for sufficiently negative values of $\alpha$. In such a case, it proves necessary to introduce the moment with a cutoff

$$
M_{\alpha}(t ; \epsilon)=\int_{\epsilon}^{\infty} x^{x} c(x, t) \mathrm{d} x
$$


in order to render the negative moments finite. From equation $(8 a), M_{0}(t)$ is the total number of clusters at time $t$ and $M_{1}(t)$ is the total mass in the system. There is an indeterminacy in these definitions which stems from the fact that there are two free parameters in the scaling ansatz, namely the amplitudes of $s(t)$ and $\phi$. Without loss of generality, we choose $m_{0}=m_{1}=1$ in order to fix these two free parameters.

If the system obeys scaling, then the 'bare' and 'scaled' moments are related by

$$
M_{\alpha}(t)=s^{\alpha-1}(t) m_{x}
$$

and, in particular,

$$
s(t)=m_{0} / M_{0}(t)
$$

The latter equality states that the average size is inversely proportional to the average number of clusters, as one expects if there is a unique typical size scale in the system. The relation between $m_{\alpha}$ and $M_{\alpha}(t)$ follows solely from the ansatz (7), therefore this relation is common to both fragmentation and aggregation.

A crucial aspect of the analysis which follows is the determination of the quantitative relation between the scaled cluster size distribution, $\phi(x)$, and the corresponding moments $m_{\alpha}$. This relation is embodied by the following correspondence. First, we use scaling to compute the moments for a discrete set of equidistant $\alpha$ values. We then invoke 'smoothness', in which the form of the moments defined on the discrete set $\{\alpha\}$ is extended to continuous values of $\alpha$. Finally, the functional form of the scaling function is determined by computing the inverse Mellin transform of $m_{\alpha}$. For example, if the moments $m_{\alpha}$ are finite for $\alpha<\alpha_{c}$ and infinite for $\alpha>\alpha_{c}$, then it is interpreted that $\phi(x)$ asymptotically behaves as the power law, $\phi(x) \propto x^{-1-\alpha_{c}}$. Although this statement is not mathematically precise, the correspondence procedure is supported by available exact solutions, and it is physically plausible. If there are cases where this correspondence does not strictly apply, the comparison of the limiting forms for $m_{\alpha}$ for two models can be a valuable measure of the closeness of the corresponding $\phi(x)$. In the following treatment, we will assume that this correspondence principle is generally valid.

\section{Linear fragmentation for $\lambda>0$}

\subsection{Scaling solutions}

For linear fragmentation, straightforward substitution of equation (7a) into (1) allows one to separate the dependence on $x$ and $t$ into two scaling equations

$$
\begin{aligned}
& \omega\left[2 \phi(\xi)+\xi \phi^{\prime}(\xi)\right]=-\xi^{\dot{\lambda}} \phi(\xi)+\int_{\xi}^{\infty} \phi(\eta) \eta^{\lambda-1} b(\xi / \eta) \mathrm{d} \eta \\
& \dot{s}(t) s(t)^{-(1+\lambda)}=-\omega .
\end{aligned}
$$

Here $\xi=x / s, \omega>0$ is the separation constant, and the overdot denotes the time derivative. The value of $\omega$ depends on the normalisation which is chosen for $m_{0}$ and $m_{1}$. From equation (12), the typical cluster size has the time dependence

$$
s(t) \sim \begin{cases}t^{-1 / \lambda} & \text { for } \lambda>0 \text { and } t \rightarrow \infty \\ \mathrm{e}^{-\omega t} & \text { for } \lambda=0 \text { and } t \rightarrow \infty \\ \left(t_{\mathrm{c}}-t\right)^{1 /|\lambda|} & \text { for } \lambda<0 \text { and } t<t_{\mathrm{c}} .\end{cases}
$$


As first shown rigorously in [9], $\lambda>0$ is a necessary and sufficient condition for the validity of scaling. (We shall present a physical and simpler line of reasoning for this result below.) Therefore the prediction for $s(t)$ when $\lambda<0$ may not apply in general.

To find the asymptotic solution to equation (11), we convert it to a relation involving the moments of $\phi(\xi)$, by multiplying both sides by $\xi^{\alpha}$ and integrating over all $\xi$. (We discuss later the conditions needed for these moments to converge.) In this derivation, the second term on the right-hand side of equation (11) gives rise to the integral

$$
\int_{0}^{x} \mathrm{~d} \xi \int_{\xi}^{x} \xi^{\alpha} \eta^{i-1} \phi(\eta) g(\xi / \eta) \mathrm{d} \eta
$$

and the order of integration can be interchanged to yield

$$
\int_{0}^{\eta} \mathrm{d} \xi \int_{0}^{\infty} \xi^{x} \eta^{\lambda-1} \phi(\eta) g(\xi / \eta) \mathrm{d} \eta .
$$

By introducing the moments of the reduced breakup kernel

$$
L_{\alpha}=\int_{0}^{1} x^{\alpha} b(x) \mathrm{d} x
$$

we obtain the linear recursion relation for the moments of the scaling function, $m_{\alpha}$,

$$
m_{x+i}=\omega \frac{1-\alpha}{L_{\alpha}-1} m_{\alpha}
$$

The explicit dependence on the kernel is contained only in $L_{\alpha}$. This suggests that the results which follow from this recursion relation will be universal. From equation (16), we compute the asymptotic form of $m_{x}$ for a discrete set of equidistant $\alpha$ values and then use the properties of the inverse Mellin transform to reconstruct the functional form of the scaling function. These details are presented separately for large and small $x$.

4.1.1. Large $x$ limit. The large $x$ behaviour of $\phi(x)$ corresponds to $m_{\alpha}$ for large values of $\alpha$. To obtain these moments, choose $\alpha=k \lambda$, with $k$ a positive integer, iterate equation (16), and use $m_{0}=1$. This yields

$$
m_{k i}=\omega^{k-1} \prod_{n=1}^{k-1} \frac{n \lambda-1}{1-L_{n i}}
$$

For large $k$, the product is dominated the large- $n$ factors, and we now demonstrate that this naturally leads to a universal behaviour of the moments.

To illustrate, consider the class of kernels which have the form, for $x \rightarrow 1$,

$$
b(x)=b(1)+\mathrm{O}\left((1-x)^{\mu}\right)
$$

where $b(1) \geq 0$ and $\mu>0$ are constants. For this form of $b(x)$

$$
L_{x}=b(1) / x+\mathrm{O}\left(x^{-(\mu+1)}\right)
$$


for large $\alpha$. Using this result, together with the large- $N$ approximation, $\prod_{n=1}^{N}(1-a / n) \propto$ $N^{-a}$, in equation (17), we obtain

$$
m_{k \lambda} \propto(\omega \lambda)^{k-1}(k-1) !(k-1)^{(b(1)-1) / \lambda} .
$$

Employing Stirling's approximation yields

$$
m_{\alpha} \rightarrow c(\omega / e)^{\alpha / \lambda} \alpha^{(b(1)-1) / \lambda-1 / 2} \alpha^{\alpha / \lambda} \quad \text { for } \alpha \rightarrow \infty
$$

where $c$ is a constant and where $k$ has been replaced by $\alpha / \lambda$. Although equation (21) has been derived for a discrete set of equidistant $\alpha$ values, we expect that this equation is generally true, since $m_{*}$ is smoothly varying in $\alpha$.

In this limiting form for $m_{\alpha}$, the dependence on the breakup kernel appears only through $\lambda$ and $b(1)$. The controlling factor, $\alpha^{\alpha / \lambda}$, arises from $\prod_{n=1}^{k-1}(n \lambda-1)$, while the next-order term, $x^{(b(1)-1) / i}$, arises only from the leading behaviour of the product of $1-L_{n \hat{n}}$. The $\mathrm{O}\left((1-x)^{\mu}\right)$ term in $b(x)$ does not contribute to the leading behaviour of $m_{x}$. Owing to this universality of $m_{x}$, the corresponding functional form of $\phi(x)$ is also universal.

The general form of $\phi(x)$ is now obtained by computing the inverse Mellin transform of $m_{x}$. This gives

$$
\phi(x) \sim x^{b(1)-2} \exp \left(-a x^{\lambda}\right) \quad x \rightarrow \infty
$$

where the constant $a$ equals $1 / \lambda \omega$. It can be easily verified that this expression indeed yields the large- $\alpha$ behaviour of $m_{\alpha}$ given in equation (21). Moreover, this form for $\phi(x)$ is consistent with previously derived results for specific fragmentation models (see e.g.[11]). Thus we conclude that $\phi(x)$ has the universal controlling factor $\exp \left(-a x^{\lambda}\right)$ for large $x$, for arbitrary kernels with homogeneity index $\lambda$.

4.1.2. Small $x$ limit. In the small- $x$ limit, there is a lesser degree of universality, since the small-mass tail is not directly influenced by particles of the typical size. We do find, however, that there are only two generic forms for $\phi(x)$, whose applicability depends on whether or not the moments $L_{-x}$ exist for $\alpha \rightarrow \infty$. To obtain $\phi(x)$ in the small- $x$ limit, we require the behaviour of $m_{-x}$ as $\alpha \rightarrow \infty$. Accordingly, we choose $\alpha=1-k \lambda$ in equation (16) and iterate to arrive at the analogue of equation (17), namely

$$
m_{1-k \lambda}=\omega^{-k} \frac{\prod_{n=1}^{k}\left(L_{1-n \lambda}-1\right)}{\prod_{n=1}^{k} n \lambda} .
$$

By analogy with the case of large positive $\alpha$, the $k$ dependence of $m_{1-k i}$ for large $k$ is determined by the limiting form of $b(x)$ for $x$ near 0 .

First, consider the general situation of kernels which are cut off at small fragment sizes i.e. 'flaking off' of infinitesimal size pieces in a single breakup event is not allowed. One example is the kernel $b(x)=0$ for $x<x_{0}$, with $0<x_{0}<1$, and $b(x)=b_{1}\left(x-x_{0}\right)^{\mu}$ for $x \rightarrow x_{0}^{+}$. From equation (15), $L_{1-\alpha} \sim c x_{0}^{-\alpha} / \alpha^{1+\mu}$ for large $\alpha$, where $c$ is a constant. Substituting this leading behaviour into equation (23), yields

$$
m_{1-k i} \sim\left(\frac{c}{\omega \lambda^{\mu+2}}\right)^{k}(k !)^{-\mu-2} x_{0}^{-i(k+1) k / 2}
$$


Thus the controlling factor of $m_{\alpha}$ in the large- $\alpha$ limit is

$$
m_{-\alpha} \sim \exp \left(\frac{-\ln x_{0}}{2 \lambda} \alpha^{2}\right) \quad \alpha \rightarrow \infty
$$

with the exponent $\mu$ appearing in lower-order corrections. The inverse Mellin transform of $m_{-\alpha}$ can be obtained straightforwardly, and leads to the classical log-normal form for the controlling factor of $\phi(x)$ :

$$
\phi(x) \sim \exp \left(-\frac{\lambda}{2 \ln x_{0}}\left(\ln ^{2} x\right)\right) \quad(x \rightarrow 0) .
$$

This expression represents a strict lower bound for the small-mass limit of the cluster-size distribution. That is $\phi(x)$ decays as $\exp \left[-c\left(\ln ^{2} x\right)\right]$, or slower, as $x \rightarrow 0$, independent of the limiting form of $b(x)$ as $x \rightarrow 0$. This follows because the controlling factor of $m_{-x}$ is given by $\prod_{n=1}^{k}\left[L_{1-n \lambda}-1\right]$. For any kernel $b(x)$, there will always exist some $0<x_{0}<1$ for which $L_{-x}>x_{0}^{-\alpha}$. Therefore the product of the $L_{-\alpha}$ diverges as $\exp \left(c \alpha^{2}\right)$ or faster, as $\alpha \rightarrow \infty$, while the other factors in equation (23) are bounded by $\exp \left(c^{\prime} \alpha \ln \alpha\right)$. Consequently, $\phi(x)$ decays as $\exp \left[-c\left(\ln ^{2} x\right)\right]$, or slower, as $x \rightarrow 0$.

The log-normal form can also be obtained from a simple multiplicative argument which appears to contain the essence of a repeated fragmentation process with a small size cutoff (see e.g. [24, 25 and references therein, 26]). According to this process, the mass of a given fragment schematically evolves as

$$
x_{0} \rightarrow x_{1} \rightarrow x_{2} \rightarrow \cdots \rightarrow x_{N}
$$

where the successive reduction factor, $r_{k}=x_{k} / x_{k-1}$, is a random variable with a well behaved distribution, for a kernel with a small-mass cutoff. By the central limit theorem, $\log x_{N}=\sum_{k=0}^{N} \log r_{k}$ will be normally distributed, so that $x_{N}$ will be distributed $\log$-normally.

A second general class of behaviour arises for kernels in which flaking off of infinitesimal size pieces in a single fragmentation event is allowed. This is typified by a power-law decay in $b(x)$ for small $x$ i.e. $b(x) \sim x^{\nu}$. In this case, it follows from equation (16) that $m_{\alpha}$ diverges whenever $L_{\alpha}$ diverges. This divergence occurs for $\alpha$ less than a critical value $\alpha_{c}$, which is less than 0 , since $m_{0}$ is finite. For $\alpha$ close to $\alpha_{c}$ we keep only the leading term in equation (16) to give

$$
m_{\alpha} \simeq L_{\alpha} \frac{m_{\alpha_{c}+\lambda}}{\omega\left(1-\alpha_{c}\right)} \propto L_{\alpha} .
$$

Since $m_{x}$ is proportional to $L_{x}$, it follows that $\phi(x)$ coincides with $b(x)$. That is,

$$
\phi(x) \sim x^{y} \quad \text { as } x \rightarrow 0 .
$$

Thus for a kernel with no small-size cutoff, the limiting form of $\phi(x)$ now decays as $\exp (-v \ln x)$, which is much slower than the $\log -$ normal bound, $\exp \left[-c(\ln x)^{2}\right]$. We believe that equations (22), (26) and (29) provide the asymptotic behaviours of $\phi(x)$ for an encompassingly wide class of breakup kernels. The range of possibilities are summarised by the 'phase diagram' of figure 1 . 


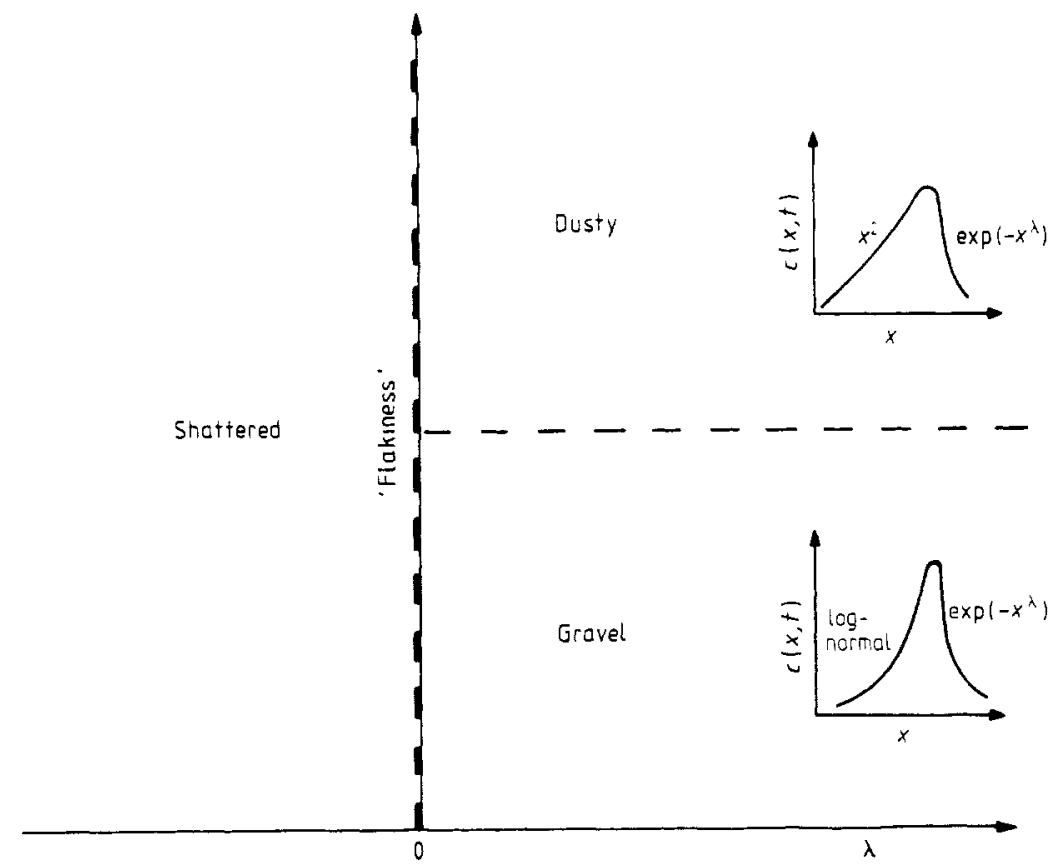

Figure 1. Phase diagram for linear fragmenta. on in the plane defined by the homogeneity index $\lambda$ and a loosely defined parameter, the "akiness' of the relative breakup rate. Small flakiness corresponds to a vanishingly small probability of small flakes being produced in a single breakup event i.e. a small-size cutoff in $b(x)$, while large flakiness corresponds to the opposite limit of a power-law tail in $b(x)$. In the phase plane there is a 'shattered' phase for $i<0$, while for $i \geq 0$ there is a 'gravel' phase for small flakiness and a 'dusty' phase for large fiakiness. The fragment size distributions corresponding to these latter two phases are sketched.

\subsection{Existence of scaling}

As mentioned above, scaling has rigorously been shown to be valid for $\lambda>0$ [9]. We now present a simpler, physical approach which shows that $\lambda>0$ is a necessary condition for scaling, and we also give a plausibility argument that $\lambda>0$ is also a sufficient condition for scaling to apply.

First we show that $\lambda<0$ implies no scaling. If scaling did hold for $\lambda<0$, then equation (13) would predict that there is a singularity in $s$ at a finite time, and by equation (10), $M_{0}(t)$ would vanish at a finite time. This contradicts the fact, from equation (1), that $c(x, t)$ must decay exponentially in time or slower, for any $x$. Hence scaling solutions do not exist when $\lambda<0$.

To argue that $\lambda>0$ is a sufficient condition for scaling, we follow steps, similar to those that led to equation (16), to derive the 'bare' moment relation

$$
\dot{M}_{\alpha}=\left(L_{\alpha}-1\right) M_{\alpha+i}
$$

Starting with $M_{1}(t)=1$, equation (30) gives $M_{1-\lambda}(t)=\left(L_{1-\lambda}-1\right) t+$ constant, where $L_{1-\lambda}-1$ is a positive constant. Iterating this process, one finds the asymptotic solution

$$
M_{1-k i} \simeq \frac{1}{k !} \prod_{j=1}^{k}\left(L_{1-j i}-1\right) t^{k}
$$


for a discrete set of equidistant index values $1-k \lambda$. Assuming that the form of $M_{\alpha}$ for arbitrary $\alpha$ interpolates smoothly between the moments defined on the discrete set, then equation (30) reproduces the temporal behaviour of the moments from the scaling ansatz.

It is worth emphasising that the negative moments of the cluster size distribution are the fundamental dynamical quantities that characterise the fragmentation process. They play a role analogous to that of the positive moments of the cluster size distribution in growth phenomena such as aggregation.

\section{Linear fragmentation for $\lambda<0$}

\subsection{Scaling theory}

For the asymptotic solution to the cluster size distribution when $\lambda<0$, we argue that a modified scaling ansatz for $\phi(x)$ may be written which superficially resembles equation $(7 a)$, except that the typical cluster size is determined by the initial condition, rather than by the dynamics of the fragmentation process.

We will justify this modified scaling ansatz by first showing that there is an anomaly in the time dependence of $c(x, t)$ for $\lambda<0$. Notice first that for the case of a monomeronly initial condition, $c(x, t=0)=\delta(x-1)$, the scaling solution for $\phi(x)$ given in equation (22) yields the time dependence, $c(x, t) \sim \mathrm{e}^{-x^{i} t}$. The coefficient of $t$ in the exponential is a decreasing function of $x$ for $\lambda>0$, so that smaller clusters decay more slowly. We now argue that for $\lambda<0, c(x, t) \sim \mathrm{e}^{-a t}$, with the coefficient $a$ 'sticking' at a value of unity. This feature is the source of the differing scaling forms for $\lambda>0$ and $\lambda<0$.

Our argument is based on showing that $\mathrm{e}^{-a t}$, with $a=1$, represents both an upper and a lower bound to the decay of $c(x, t)$. To show first that $c(x, t) \geq \mathrm{e}^{-t}$, let us assume the opposite and derive a contradiction. An obvious lower bound for $c(x, t)$ is attained by neglecting the gain terms in equation (1), leading to $c(x, t)$ decaying as $\mathrm{e}^{-x^{i} t}$. Since $\lambda<0$, faster decay would occur for smaller values of $x$, so that $c(1, t)=\mathrm{e}^{-t}$ is the slowest decay mode in the system. This slowest mode feeds in to the gain term in equation (1) via $c(1, t)$. Now if $c(x, t)$, and hence the loss term, were to decay faster than $\mathrm{e}^{-t}$, then the gain term will eventually dominate. This would lead to $\dot{c}(x, t)>0$, which contradicts our original assumption. Therefore we conclude that $c(x, t) \geq e^{-t}$. Now we also show that $c(x, t)$ cannot decay slower than $\mathrm{e}^{-t}$. Assuming the opposite, then for some $x<1$ the loss term in equation (1) will dominate. However, if the gain term in equation (1) is negligible, there would be a faster than $\mathrm{e}^{-t}$ decay for $c(x, t)$, invalidating the assumption that $c(x, t) \geq \mathrm{e}^{-t}$. Thus we conclude that the controlling time dependence of $c(x, t)$ is $\mathrm{e}^{-t}$ for any $x$ in the long time limit.

Notice that the initial condition sets the timescale of the bound for $c(x, t)$ and this can be generalised to an arbitrary initial condition. If $g(x, t)$ is the Green function for the initial condition $\delta(x-1)$, then one can verify that $l^{-1} g\left(x / l, t l^{\lambda}\right)$ is the Green function for the initial condition $\delta(x-l)$, and that

$$
c(x, t)=\int_{0}^{x} \mathrm{~d} l f(l) l^{-1} g\left(x / l, t l^{\dot{x}}\right)
$$

is the solution for an arbitrary initial condition $c(x, t=0)=f(x)$. Consequently, the time dependence, $c(x, t) \sim \mathrm{e}^{-t}$, for the monomer-only initial condition translates to 
the asymptotic time dependence $c(x, t) \sim \exp \left(-l^{-|\lambda|} t\right)$ for an arbitrary initial condition $c(x, t=0)=\delta(x-l)$.

On this basis, and also by comparison with available exact solutions [12], we now propose the following ansatz for the long-time behaviour of $c(x, t)$ in the $\lambda<0$ regime, for $c(x, t=0)=\delta(x-l)$ :

$$
c(x, t)=T\left(t l^{-|\lambda|}\right) \phi(x / l) .
$$

This resembles the conventional scaling ansatz, except that $s(t)$ is replaced by $l$ in the scaling function $\phi$. In the original scaling ansatz, the mass and time dependence in the cluster size distribution is separated as $c(x, t)=T(t) \phi(x / s(t))$, in the long-time limit, by the use of an appropriate scaled mass variable. For $\lambda>0$, one of the separation equations yields the time dependence of the typical mass $s(t)$, and mass conservation fixes $T(t)$ to be $s^{-2}(t)$. In contrast, for $\lambda<0$, the initial mass, $l$, plays the role of a (time-independent) typical mass. Since mass is not conserved for $\lambda<0$, a direct examination of the rate equations is needed in order to determine the form of $T(t)$.

We now show that $\phi(x)$ has the universal form $x^{|\lambda|-2}$. By the properties of the Mellin transform, this is equivalent to showing that there exists an $\alpha_{c}=1-|\lambda|$ such that $M_{\alpha}=\infty$ for $\alpha<\alpha_{c}$ and $M_{\alpha}<\infty$ for $\alpha>\alpha_{c}$. We show this by demonstrating that $1-|\lambda|$ represents both an upper and a lower bound for the value of $\alpha_{\mathrm{c}}$. First, to show that $1-|\lambda| \leq \alpha_{\mathfrak{c}}$, we use the result from the next section that $M_{1-|\lambda|}$ is infinite. Together with the fact that $M_{\alpha}$ is a non-increasing function of $\alpha$, we conclude that there exists an $\alpha_{c}$ which satisfies $1-|\lambda| \leq \alpha_{\mathrm{c}}$. Since $M_{1}$ is a decreasing function of time, one also has the trivial bound $\alpha_{c} \leq 1$. To show that $1-|\lambda| \geq \alpha_{c}$, we write a moment relation analogous to equation (30) for the cut off moments $M_{x}(t ; \epsilon)$ introduced in equation (8b)

$$
M_{\alpha}(\epsilon)=-\frac{\dot{M}_{\alpha+|\lambda|}(\epsilon)}{1-\int_{0}^{1} \mathrm{~d} y b(y) y^{\alpha+|\lambda|} M_{\alpha}(\epsilon / y) / M_{\alpha}(\epsilon)} .
$$

Equation (34) is valid for any $\alpha$ and $\epsilon>0$, and it reduces to equation (30) if $\lim _{\epsilon \rightarrow 0} M_{\alpha}(\epsilon / y) / M_{\alpha}(\epsilon)=1$ for $0<y \leq 1$. This limiting behaviour occurs whenever $M_{\alpha}(\epsilon=0)$ is finite.

Consider this generalised moment relation for arbitrary $\alpha>1-|\hat{\lambda}|$. Since $M_{x}(\epsilon / y) / M_{x}(\epsilon) \leq 1$ for $0<y<1$, the denominator in equation (34) can be bounded from below by

$$
1-\int_{0}^{1} \mathrm{~d} y b(y) y^{z+\lambda i} \frac{M_{\alpha}(\epsilon / y)}{M_{\alpha}(\epsilon)} \geq 1-\int_{0}^{1} \mathrm{~d} y b(y) y^{\alpha+|\lambda|}>0 .
$$

(Recall from subsection 2.1 that $\int_{0}^{1} \mathrm{~d} y y^{\beta} b(y)<1$ for $\beta>1$.) Thus the limit of $\epsilon \rightarrow 0$ in equation (34) is well behaved, so that $M_{\alpha}=\lim _{\epsilon \rightarrow 0} M_{x}(\epsilon)$ must be finite. This shows that $\alpha_{c} \leq 1-|\lambda|$, and we therefore conclude that $\alpha_{c}=1-|\hat{\lambda}|$. Since our discussion has been independent of (33), we have therefore justified the validity of this modified scaling ansatz in the long-time, small-mass limit, and have also obtained the leading behaviour

$$
c(x, t) \sim \mathrm{e}^{-t} x^{|\hat{\mid}|-2} .
$$

This result has a simple physical interpretation which clarifies the role of the initial mass as the characteristic scale in the system. By invoking the scaling ansatz (33), one 
may write $M_{\alpha}(\epsilon)$ as the product $f(t) m_{\alpha}$, where $m_{\alpha}$ is time independent. Substituting this in equation (34), taking $\epsilon=0$, and $\alpha \approx 1-|\lambda|$, reduces (34) to

$$
m_{\alpha-|\lambda|} \simeq \frac{1}{1-L_{\alpha}} \quad(\alpha \gtrsim 1-|\lambda|)
$$

On the other hand, if one includes a constant source at $x=1$ in the rate equations, then the corresponding moment relation is identical to equation (37). The physical interpretation of this coincidence between the the irreversible and steady-state solutions is that when $\lambda<0$, the reaction between small clusters is so rapid that the initial mass distribution is 'left behind'. This residue reacts at a negligible rate asymptotically, and thus plays the role of a constant source in the ensuing fragmentation process.

\subsection{Shattering transition}

An intriguing aspect of fragmentation processes is the possibility of a 'shattering' transition, in which mass is 'lost' to a phase of zero-mass particles. This transition occurs when very small clusters break up sufficiently rapidly to dominate the entire fragmentation process asymptotically. This is reminiscent of gelation, where mass within the population of finite-size particles is lost to an infinite gel molecule by the very rapid aggregation of large molecules. Both gelation and shattering are signalled by the condition, $\dot{M}_{1}<0$. As first shown generally by Filippov [9] and discussed by McGrady and Ziff [12] for specific fragmentation models, shattering occurs when $\lambda<0$.

We now give a simple general argument which shows that $\lambda<0$ is the necessary and sufficient condition for shattering in a homogeneous fragmenting system. (For a general non-homogeneous system, Filippov has written a condition analogous to $\lambda<0$ as the necessary and sufficient condition for shattering.) To locate the shattering transition, we write equation (30) for $\alpha=1+\epsilon$

$$
\dot{M}_{1}=\left(L_{1+\epsilon}-1\right) M_{\lambda+1+\epsilon} .
$$

As $\epsilon \rightarrow 0^{+}, L_{\alpha}$ approaches 1 from below (mass conservation), so that $\dot{M}_{1}$ can be non-zero only if $M_{\lambda+1+\epsilon}$ diverges as $\epsilon \rightarrow 0$. Without loss of generality, suppose that the largest initial cluster mass is unity, so that will be no cluster with a mass larger than unity for $t>0$. Then $M_{\alpha}$ is non-decreasing as $\alpha$ decreases, at any fixed time. This fact, together with $M_{1}<\infty$, implies that $M_{\alpha}$ can diverge only for $\alpha<1$. Thus a necessary condition for shattering is $\lambda<0$.

To show that $\lambda<0$ is also a sufficient condition for shattering, let us assume the converse and derive a contradiction. For $\lambda<0$, equation (30) gives

$$
\dot{M}_{1+|\lambda|}=\left(L_{1+|\lambda|}-1\right) M_{1} \text {. }
$$

Under the assumption of no shattering, $M_{1}$ remains fixed, so that the right-hand side is a negative constant. This implies that $M_{1+|\lambda|}$, and consequently $c(x, t)$ would vanish at a finite time. On the other hand, from equation (1), $c(x, t)$ must decay exponentially in time, or slower, for any value of $x$. This contradiction implies that a sufficient condition for shattering is $\lambda>0$.

A characteristic feature of shattering for linear fragmentation is that the mass loss starts immediately at $t=0$. This is in contrast to the complementary situation of gelation, where mass loss does not occur until a non-zero finite gelation time. 


\section{Scaling solutions for collision-induced fragmentation}

For collision-induced fragmentation, we anticipate that the cluster size distribution will evolve into a scaling form in the long-time limit, for general homogeneous reaction kernels. Thus substituting the scaling ansatz in equation (2), one arrives at the scaling equations for $\phi(x)$ and for the typical fragment size

$$
\begin{aligned}
& \begin{aligned}
\omega\left[\xi \phi^{\prime}(\xi)+\right. & 2 \phi(\xi)] \\
& =-\phi(\xi) \int_{0}^{x} K(\xi, \eta) \phi(\eta) \mathrm{d} \eta+\int_{0}^{x} \mathrm{~d} \zeta \int_{\xi}^{x} K(\eta, \zeta) B(\xi \mid \eta, \zeta) \phi(\eta) \phi(\zeta) \mathrm{d} \eta
\end{aligned} \\
& \dot{s}(t) s(t)^{-i}=\omega^{-1}
\end{aligned}
$$

where $\omega$ is a separation constant. The solution for $s(t)$ is

$$
s(t) \sim \begin{cases}t^{1 /(1-\lambda)} & \text { for } \lambda>1 \text { and } t \rightarrow \infty \\ \mathrm{e}^{-\omega t} & \text { for } \lambda=1 \text { and } t \rightarrow \infty \\ \left(t_{\mathrm{c}}-t\right)^{1 /(\lambda-1)} & \text { for } \lambda<1 \text { and } t<t_{\mathrm{c}} .\end{cases}
$$

These behaviours are valid only if scaling holds. From equation (42), shattering is anticipated when $\lambda<\lambda_{\mathrm{s}}=1$. In contrast to linear fragmentation, the critical value of $\lambda_{\mathrm{s}}$ is unity, corresponding to a situation where smaller particles are relatively less likely to collide. This diminished small-particle collision rate is more than compensated, however, by their rapid production by two-body collisions.

To understand the qualitative features of nonlinear fragmentation, we turn to the splitting models defined in equations (4)-(6). For a monomer-only initial condition, we write the mass, $x$, as $x=2^{-n}$ with integer $n$ to simplify the form of the rate equations. We will focus on asymptotic solutions to the resulting rate equations in the scaling regime. Numerically, this obtains when $\lambda>\lambda_{c}$, where $\lambda_{c}$ is model dependent and less than one in general.

Before presenting mathematical details, we summarise our primary results graphically (figure 2). The basic classifying feature is whether reactions of small fragments with large fragments dominates over, or is dominated by, small-small reactions. (In any case, large-large reactions are irrelevant asymptotically.) In models I and II, the larger of the two colliding fragments always splits. Therefore large-small reactions eventually dominate, since large clusters become effectively the most reactive as the system evolves. As we shall derive, this yields a peaked fragment size distribution, as illustrated in the phase diagram for collision-induced fragmentation (figure $2(a)$ ). On the other hand, in model III, small-small reactions eventually dominate. Consequently large clusters become 'frozen out' of the fragmentation process at long times, and are left behind as an appreciable residue. These qualitative features have strong parallels with the corresponding phase diagram of aggregation processes (figure $2(b)$ ).

\subsection{Model I. Both particles split upon collision}

With the homogeneous collision kernel $K(x, y)=(x y)^{\lambda / 2}$ and the breakup kernel $B(x \mid y, z)=2 \delta(x-y / 2)$, the rate equations become

$$
\frac{\mathrm{d} c_{n}(t)}{\mathrm{d} t}=\left(-2^{-i n / 2} c_{n}(t)+2 \times 2^{-\lambda(n-1) / 2} c_{n-1}(t)\right) \sum_{j=0}^{\infty} 2^{-\lambda j / 2} c_{j}(t)
$$




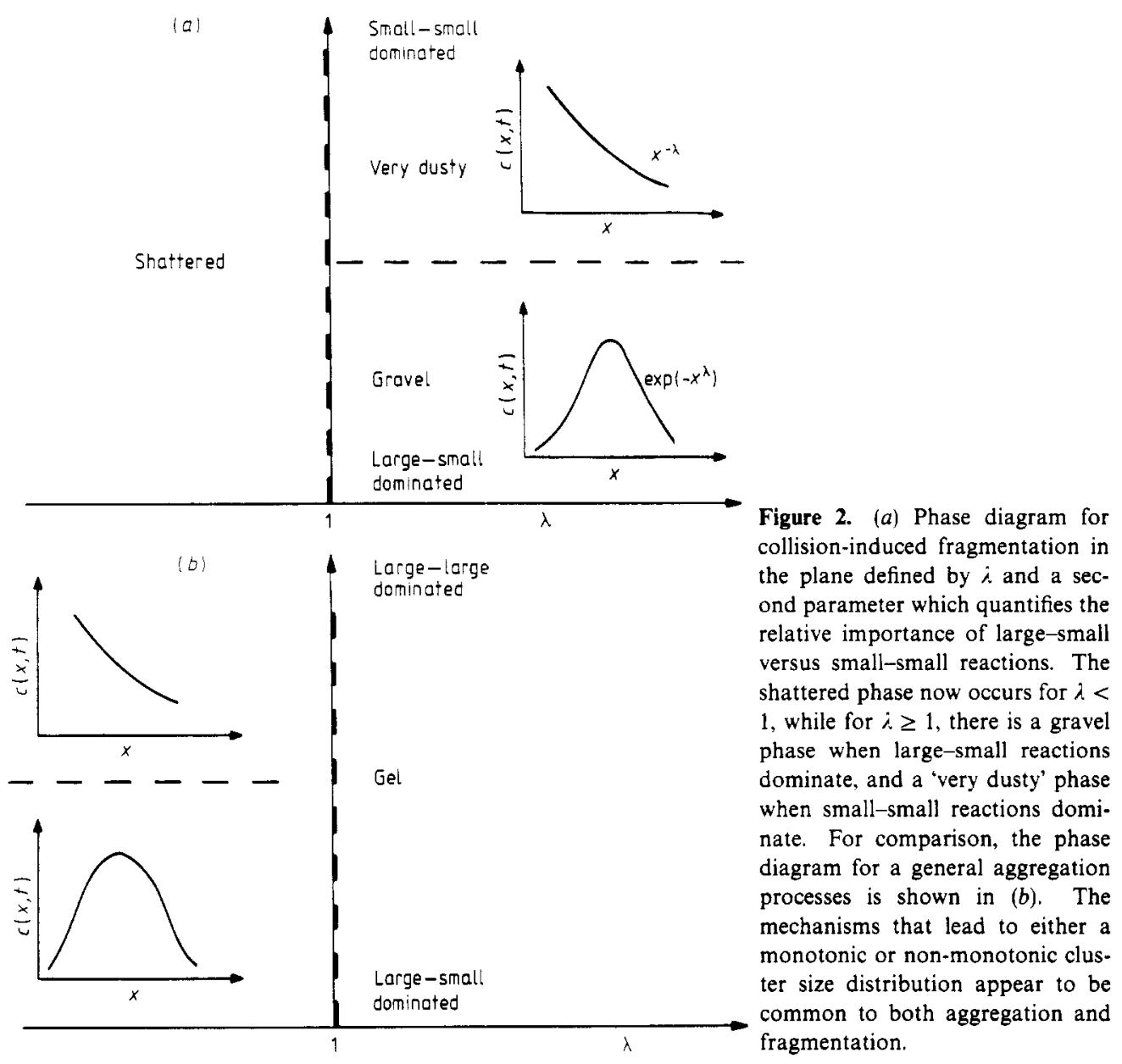

Of some pedagogical interest is the case $\lambda=0$, where both colliding clusters split at a mass-independent rate. For the initial condition $c_{n}(0)=M \delta_{n, 0}$, the exact solution for this model is

$$
c_{n}(t)=\frac{1}{n !} \frac{t_{\mathrm{c}}-t}{t_{\mathrm{c}}^{2}}\left(2 \ln \frac{t_{\mathrm{c}}}{t_{\mathrm{c}}-t}\right)^{n}
$$

where $t_{\mathrm{c}}$ is determined from the initial conditions via $t_{\mathrm{c}}=1 / M$. Mass is conserved for $t<t_{\mathrm{c}}$, but the cluster concentrations of all sizes vanish at $t_{\mathrm{c}}$.

Model I can be mapped to a linear fragmentation process by defining the rescaled time variable

$$
\tau=\int^{t} \mathrm{~d} t^{\prime} \sum_{j=0}^{\infty} 2^{-\lambda j / 2} c_{j}\left(t^{\prime}\right)=\int^{t} \mathrm{~d} t^{\prime} M_{\lambda / 2}\left(t^{\prime}\right)
$$


which leads to the linear rate equations

$$
\frac{\mathrm{d} c_{n}(\tau)}{\mathrm{d} \tau}=-2^{-i n / 2} c_{n}(\tau)+2 \times 2^{-i(n-1) / 2} c_{n-1}(\tau)
$$

This transformation is well behaved only for $\dot{\lambda}>0$. As a result, model $\mathrm{I}$ is not defined for $\lambda<0$. In this mapping, the parameters that characterise the breakup kernel in the linear model are related to those of the corresponding nonlinear model by $\lambda_{\text {linear }}=(\lambda / 2)_{\text {nonlinear }}, b(x)_{\text {linear }}=2 \delta(x-1 / 2)$. From previous results for the linear model, we therefore conclude that scaling is valid for $\lambda>0$, and that shattering occurs for $\lambda<1$. This shattering transition is of a different character than that in the linear model, as mass loss does not occur until a finite shattering time $t_{\mathrm{c}}$.

In the scaling regime $(\lambda>0)$, we exploit the mapping to the linear model to write the asymptotic form of the cluster size distribution for model I

$$
\phi(x) \sim \begin{cases}\exp \left(-x^{\lambda / 2}\right) / x^{2} & x \rightarrow \infty \\ \exp \left(\frac{\lambda}{4 \ln 2}\left(\ln ^{2} x\right)\right) & x \rightarrow 0 .\end{cases}
$$

Here $x=2^{-n} / s(t)$, and $s(t)$ is given by equation (42). From the scaling ansatz, the following limiting behaviour for $c_{n}(t)$ can be deduced:

$$
c_{n}(t) \sim \begin{cases}2^{n} \exp \left[-\left(\frac{2^{-n}}{s(t)}\right)^{\lambda / 2}\right] & s(t) \ll 2^{-n} \\ s(t)^{-1} \exp \left[\frac{\lambda}{4 \ln 2} \ln ^{2}\left(2^{-n} / s(t)\right)\right] & s(t) \gg 2^{-n} .\end{cases}
$$

\subsection{Model II. Larger particle splits upon collision}

Substituting the kernels (5) into equation (2) gives the rate equations

$$
\begin{aligned}
\frac{\mathrm{d} c_{n}}{\mathrm{~d} t} & =-2^{-\lambda n} c_{n} \sum_{j=n}^{\infty} c_{j}+2 \times 2^{-i(n-1)} c_{n-1} \sum_{j=n-1}^{\infty} c_{j} \\
& \equiv-\gamma^{n} c_{n} \sum_{j=n}^{\infty} c_{j}+2 \gamma^{n-1} c_{n-1} \sum_{j=n-1}^{\infty} c_{j}
\end{aligned}
$$

where $\gamma=2^{-\lambda}$. In the long-time limit, $c_{n}$ decays to zero for any fixed $n$, while the total number of clusters increases indefinitely. Consequently the lower limits on the sums in equation (48) can be extended to 0 , leading to equations which are very similar to equation (43) of model 1 . Linearising the resulting equations by defining the rescaled time variable $\tau=\int^{t} \mathrm{~d} t^{\prime} M_{0}\left(t^{\prime}\right)$ gives

$$
\frac{\mathrm{d} c_{n}(\tau)}{\mathrm{d} \tau}=-\gamma^{n} c_{n}(\tau)+2 \gamma^{n-1} c_{n-1}(\tau)
$$

By this device, model II is now mapped to a linear fragmentation problem whose behaviour is known, in principle. 
However, we give a more direct approach which also serves as a check of the scaling ansatz for model II. By elementary methods, the solution to equation (49) can be written as

$$
c_{n}(\tau)=\mathrm{e}^{-\gamma^{n} \tau}\left(a_{n}-2 \gamma^{n-1} \int_{\tau}^{\infty} \mathrm{d} \tau^{\prime} \mathrm{e}^{\gamma^{n} \tau^{\prime}} c_{n-1}\left(\tau^{\prime}\right)\right)
$$

where

$$
a_{n}=c_{n}(0)+2 \gamma^{n-1} \int_{0}^{x} \mathrm{~d} \tau^{\prime} \mathrm{e}^{\gamma^{n} \tau^{\prime}} c_{n-1}\left(\tau^{\prime}\right)
$$

is positive. We have used the boundary condition that all the cluster concentrations of finite mass are equal to zero at infinite time. One reason for specifying the boundary condition in this manner is that the contribution of the integral in equation $(50 a)$ becomes vanishingly small as $\tau \rightarrow \infty$, and this facilitates the construction of the asymptotic solution. Since $c_{n}(\tau)$ is non-negative for any $n$, equation (50a) provides the upper bound

$$
c_{n}(\tau) \leq a_{n} \mathrm{e}^{-\gamma^{n} \tau}
$$

Employing this bound for $c_{n-1}$ in equation (50a) gives the lower bound $c_{n}(\tau) \geq a_{n} \mathrm{e}^{-\gamma^{n} \tau}$. Thus we conclude that for $\lambda>0$ and large $\tau$

$$
c_{n}(\tau) \sim a_{n} \mathrm{e}^{-\gamma^{n} \tau}
$$

The amplitude $a_{n}$ cannot be determined merely by substituting equation (52) into $(50 b)$, since equation (52) is valid only for large $\tau$. However, since the recursion relation for $a_{n}$ involves the Laplace transform of $c_{n-1}(\tau)$, we define the Laplace transform $\tilde{c}_{n}(\sigma)=\int_{0}^{\infty} d \tau c_{n}(\tau) \mathrm{e}^{-\sigma \tau}$, to reduce equations $(49)$ and $(50 b)$ to the linear recursion relations

$$
\begin{aligned}
& \tilde{c}_{n}(\sigma)=\frac{c_{n}(0)+2 \gamma^{n-1} \tilde{c}_{n-1}(\sigma)}{\sigma+\gamma^{n}} \\
& a_{n}=c_{n}(0)+2 \gamma^{n-1} \tilde{c}_{n-1}\left(-\gamma^{n}\right) .
\end{aligned}
$$

For a monomer-only initial condition, the leading contribution to $\tilde{c}_{n-1}\left(-\gamma^{n}\right)$ varies as $(2 / \gamma)^{n-1}$, for $\lambda>0$ and for large $n$. Consequently equation (53b) gives $a_{n} \propto 2^{n}$, from which

$$
c_{n}(t) \sim 2^{n} \exp \left(-2^{-i n} \tau(t)\right) \quad \tau \rightarrow \infty
$$

Up to an overall constant factor this asymptotic solution is independent of the initial condition. We now determine $\tau(t)$ by noting that scaling gives $M_{0}(t) \sim 1 / s(t)$. Using the time dependence for $s(t)$ given in equation (42), $\tau=\int^{t} M_{0}\left(t^{\prime}\right) \mathrm{d} t^{\prime}$ can be identified with $s(t)^{-\lambda}$. Thus we can express $c_{n}(t)$ in the scaling form

$$
c_{n}(t) \sim 2^{n} \exp \left[-\left(\frac{2^{-n}}{s(t)}\right)^{i}\right]
$$


in the long-time limit. Since equation $(55 a)$ is obtained independent of the scaling ansatz, we have shown its validity at large $x$ when $\lambda>0$. Numerical simulations also indicate that scaling is valid for all $x$ when $\lambda>0$.

To find the small-mass (large- $n$ ) behaviour of $c_{n}(t)$, we have numerically integrated the rate equations. This calculation indicates that $c_{n}(t)$ has a controlling factor $\exp \left(-c / 2^{-n}\right)$ for some positive $\lambda$-dependent constant $c$. We can justify this form through an analysis of the scaling equation. Substituting kernels (5) into equation (40) yields

$$
\omega \frac{\left[x^{2} \phi(x)\right]^{\prime}}{x}=4(2 x)^{\dot{\lambda}} \phi(2 x) \int_{0}^{2 x} \phi(\eta) \mathrm{d} \eta-x^{\lambda} \phi(x) \int_{0}^{x} \phi(\eta) \mathrm{d} \eta .
$$

Since in model II only the larger particle breaks in a collision, we anticipate that $\phi(x)_{\text {modelII }} \leq \phi(x)_{\text {modelI }}$ in the limit $x \rightarrow 0$. Thus $\phi(x)$ decays faster than any power law for small $x$. Consequently, we can neglect the loss term in equation (56) and determine the small- $x$ behaviour of $\phi(x)$ by the gain term. If there is a faster-than-power-law decay in $\phi(x)$, differentiation or integration does not alter the asymptotic behaviour of the controlling factor. Therefore this factor satisfies

$$
\phi(x)=\phi^{2}(2 x) \quad x \rightarrow 0
$$

with the condition $\phi(0)=0$. This has the solution $\exp (-c / x)$, in agreement with our numerical calculations.

Recasting equation (55a) in terms of scaling and combining this with our result for small $x$ limit, we have

$$
\phi(x) \sim \begin{cases}\exp \left(-c_{1} x^{i}\right) / x^{2} & x \rightarrow \infty \\ \exp \left(-c_{2} / x\right) & x \rightarrow 0\end{cases}
$$

where $x=2^{-n} / s(t), c_{1}$ and $c_{2}$ are positive constants, and $s(t)$ is determined by equation (42).

\subsection{Model III. Smaller particle splits upon collision}

For a homogeneous system, the rate equations for model III are

$$
\frac{\mathrm{d} c_{n}(t)}{\mathrm{d} t}=-\gamma^{n} c_{n} \sum_{j=0}^{n} c_{j}+2 \gamma^{n-1} c_{n-1} \sum_{j=0}^{n-1} c_{j}
$$

The rate equation for $c_{0}(t)$ immediately leads to the asymptotic solution

$$
c_{0}(t) \sim 1 / t
$$

We now exploit this result to find the long-time behaviour of $c_{n}(t)$. First, define the variables

$$
\begin{aligned}
& R_{n}(\tau)=c_{n}(t) / c_{0}(t) \\
& \tau=\int^{t} \mathrm{~d} t^{\prime} c_{0}\left(t^{\prime}\right) \sim \ln t
\end{aligned}
$$


and recast equation $(58)$ as

$$
\frac{\mathrm{d} R_{n}(\tau)}{\mathrm{d} \tau}=-\gamma^{n}\left(R_{n}^{2}(\tau)-A_{n}(\tau) R_{n}(\tau)-B_{n}(\tau)\right) \quad n>0
$$

where

$$
\begin{aligned}
& A_{n}(\tau)=\gamma^{-n}-\sum_{j=0}^{n-1} R_{j}(\tau) \\
& B_{n}(\tau)=2 \gamma^{-1} R_{n-1}(\tau) \sum_{j=0}^{n-1} R_{j}(\tau) .
\end{aligned}
$$

Since $A_{n}(\tau)$ and $B_{n}(\tau)$ involve only the $R_{j}(\tau)$ with $j<n$, it is not difficult to show inductively that the ratio $R_{n}(\tau)$ converges to a positive constant $r_{n}$ for any $n$ in the long-time limit. Thus we conclude that

$$
c_{n}(t) \sim r_{n} / t
$$

where $r_{n}$ is determined iteratively by

$$
\begin{aligned}
& r_{n}=\frac{1}{2}\left(\sqrt{a_{n}^{2}+4 b_{n}}+a_{n}\right) \\
& a_{n}=\gamma^{-n}-\sum_{j=0}^{n-1} r_{j} \quad b_{n}=2 \gamma^{-1} r_{n-1} \sum_{j=0}^{n-1} r_{j} .
\end{aligned}
$$

Here $a_{n}=A_{n}(\tau=\infty)$ and $b_{n}=B_{n}(\tau=\infty)$.

To analyse these recursion relations, recall that if scaling holds, then $c_{n}(t) \sim r_{n} / t$ can be cast in the discrete-mass scaling form, $s^{-1} \phi(x / s)$. This implies that $r_{n} / \gamma^{-n}$ approaches a finite positive constant for $n \rightarrow \infty$. Consequently, it is natural to introduce two 'dimensionless' variables

$$
z_{n}=\gamma^{n} r_{n} \quad s_{n}=\gamma^{n+1} \sum_{i=0}^{n} r_{i}
$$

in terms of which equation (64) can be rewritten as

$$
\begin{aligned}
& z_{n+1}=\frac{1}{2}\left[\sqrt{8 z_{n} s_{n}+\left(1-s_{n}\right)^{2}}+\left(1-s_{n}\right)\right] \\
& s_{n+1}=\gamma\left[z_{n+1}+s_{n}\right] .
\end{aligned}
$$

The validity of scaling is equivalent to the existence of a stable finite fixed point for $z_{n}$ and $s_{n}$. For $\lambda>1$, equation (66) has two fixed points. A stable fixed point is located at

$$
z^{(1)}=(1-\gamma) /(1-2 \gamma) \quad s^{(1)}=\gamma /(1-2 \gamma)
$$

while an unstable fixed point is located at

$$
z^{(2)}=+\infty \quad s^{(2)}=+\infty
$$


For $\lambda<1$, only the second fixed point exists, and we need to determine the rate at which this fixed point is approached in order to calculate $z_{n}$. For large $z_{n}$ and large $s_{n}$, equation (66) can be approximated by

$$
\begin{aligned}
& z_{n+1}=z_{n}\left(\frac{1}{2 v_{n}}\left(\sqrt{1+8 v_{n}}-1\right)\right) \\
& v_{n+1}=\gamma^{-1} \frac{\sqrt{1+8 v_{n}}-1}{\sqrt{1+8 v_{n}}+1}
\end{aligned}
$$

where we have defined $v_{n}=z_{n} / s_{n}$. Now there are two fixed points for $v_{n}$, namely, $v^{(1)}=\gamma^{-1}(1-\sqrt{\gamma / 2})$, which is the attractor for $\lambda>-1$, and $v^{(2)}=0$, which is the attractor otherwise. Thus $z_{n}$ increases as $2^{n(1-i) / 2}$, or as $2^{n}$ for these two cases, respectively. Accordingly, the asymptotic large-time solution for $c_{n}(t)$ is

$$
c_{n}(t) \sim \begin{cases}2^{n \lambda} / t & \lambda \geq 1 \\ 2^{n(1+\lambda) / 2} / t & 1>\lambda \geq-1 \\ 2^{n(1+\lambda)} / t & -1>\lambda .\end{cases}
$$

We see that scaling breaks down for $\lambda<1$ in the large-time limit. Thus for model III, the two ranges of $\lambda$ for which scaling breaks down and for which shattering occurs, coincide.

From the above analysis, the asymptotic behaviour of $c_{n}(t)$ results results from balancing the terms on the right-hand side of equation (61); neither the gain nor the loss term can be neglected. This is in contrast to models I and II, where the loss term eventually dominates. This situation is similar to linear fragmentation for $\lambda<0$, as the decay rate of the initial monomer sets the time dependence for any other mass. This is because the initial monomer has the slowest decay mode, and it acts like a steady source for small mass.

The properties of model III in the large- $n$ limit can again be obtained by mapping the system to a linear model. In the large- $n$ limit, the upper bounds of the sums in equation (58) can be replaced by $\infty$. We can then linearise the rate equations by the same procedure used in models I and II. By this mapping, we also infer that scaling holds for $\lambda>0$ when $n \rightarrow \infty$. Since scaling holds for $\lambda>1$ in the large- $t$ limit, and for $\lambda>0$ in the large- $n$ limit, we expect that scaling holds generally for $\lambda>1$. The asymptotic form of the scaling function is

$$
\phi(x) \sim \begin{cases}x^{-(1+\lambda)} & x \rightarrow \infty \\ \exp \left(-\frac{\lambda}{2 \ln 2}\left(\ln ^{2} x\right)\right) & x \rightarrow 0\end{cases}
$$

where $x=2^{-n} / s(t)$.

\section{Numerical integration of the rate equations}

For the three splitting models, we have performed numerical integration of the rate equations, with the initial condition $c_{n}(t=0)=\delta_{n, 0}$. From these calculations we 
determine the range of values for $\lambda$ which lead to scaling and to shattering for the splitting models, and verify the asymptotic behaviours of the scaling functions.

To determine the range of $\lambda$ for which shattering occurs, we study the time dependence of the total number of fragments, $M_{0}(t)$ (figures $3(a-c)$ ). It does appear that $M_{0}(t)$ grows at a power-law rate for $\lambda>1$ and diverges in a finite time whenever $\lambda<1$, indicating that $\lambda=1$ delineates the shattering transition. Furthermore, equation (42) predicts that if $\lambda=1$ is in the scaling regime, then $M_{0}(t)=s^{-1}(t)$ grows exponentially in time. This point is investigated in figure $3(d)$. For models I and II, it appears that $M_{0}(t)$ does grow exponentially. For model III, scaling does not hold for $\lambda=1$ and $M_{0}(t)$ grows more slowly.

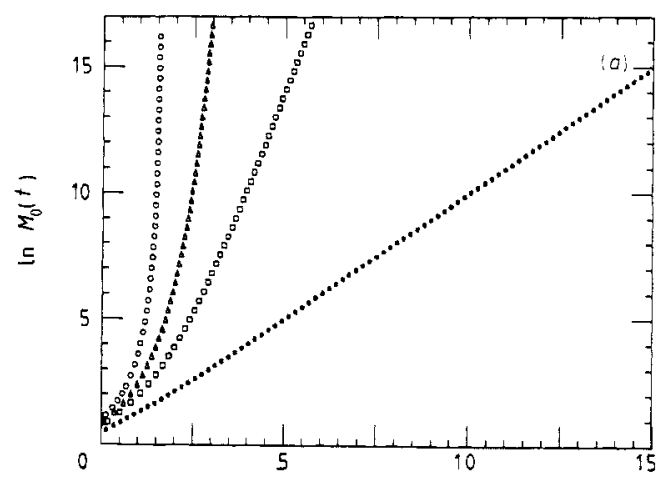

$\ln t$

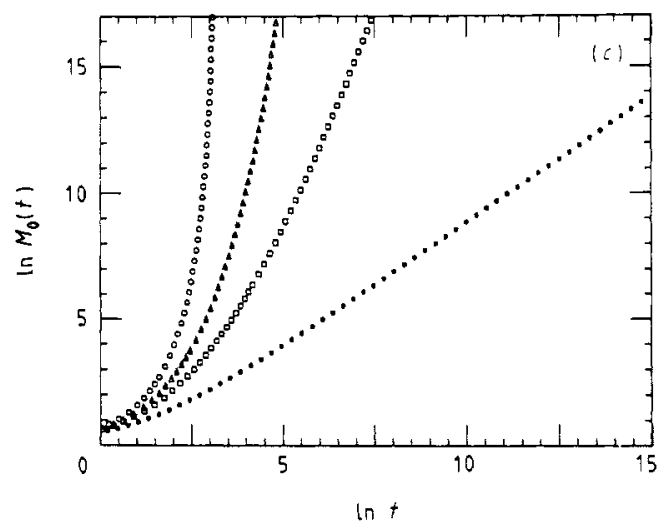

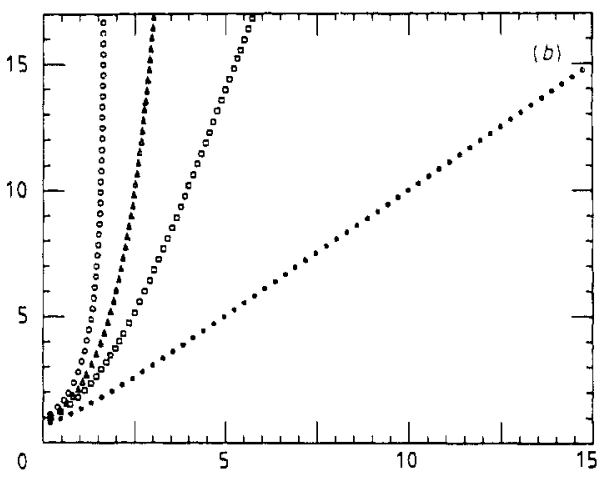

$\ln t$

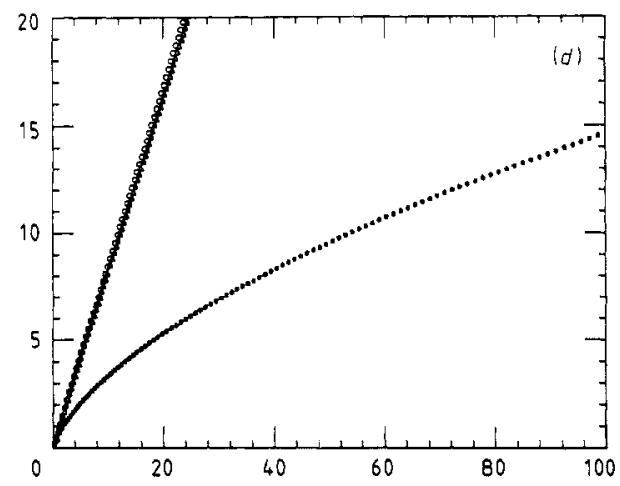

Figure 3. Plots of total number of clusters, $M_{0}(t)$, against time $t$ for models I, II, and III on a double logarithmic scale, for various values of $\lambda$ in $(a)-(c)$, respectively. Shown are the cases $\lambda=0.75(0), \lambda=1.0(\Delta), \lambda=1.25(\square), \lambda=2.0($.$) . In (d), we plot \ln M_{0}(t)$ against $t$ on a linear scale for models I-III $(\Delta, 0$, and ., respectively) for the case $\lambda=1$. In models I and II, the linear behaviour indicates that $M_{0}(t) \sim \mathrm{e}^{c t}$ for the three models.

Next, we test the scaling form for the cluster size distribution for various values of $\lambda$ by plotting the scaled concentration $\left(s(t)^{2} / x\right) c_{n}(t)$ against $x / s(t)$, where $x=2^{-n}$ is the cluster mass (figure 4). For each value of $\lambda$, selected data for $c_{n}(t)$ with $n$ ranging between 2 and 100 are taken, for approximately 100 time values $t_{i}$. The $t_{i}$ were chosen to keep the ratio $M_{0}\left(t_{i}\right) / M_{0}\left(t_{i+1}\right)$ approximately a constant, independent of $i$. This leads to $M_{0}(t)$ ranging between $10^{2}$ to $10^{9}$. In general, the particle size distributions for 
various times can be collapsed onto a single universal curve when $\lambda>1$. However, for smaller values of $\lambda$, the scaling function becomes progressively sharper, and the quality of the data collapsing gradually deteriorates until eventually the scaling form breaks down. This breakdown of scaling appears to occur when $\lambda \leqslant 0$ for models 1 and II, and when $\lambda \lesssim 1$ for model III.

Finally, we present in figure 5 the asymptotic limits of the cluster size distributions given in equations (47), (55) and (69). We generated data for the cases $\lambda=1.5,2.0$ and 2.5 , which is well within the scaling regime for all three splitting models. In order to isolate the large-mass behaviour of the distribution, we plot $\ln \left|\ln \left[\phi(x) x^{2}\right]\right|$ against $\ln x$ for models I and II (figures $5(a)$ and $(b)$, respectively), and $\ln \phi(x)$ against $\ln x$ for model III (figure $5(c)$ ). These data are well fitted by straight lines of slope $\lambda / 2$ in $(a), \lambda$ in $(b)$, and $\lambda+1$ in $(c)$, in accord with our theoretical predictions. Analogous graphical results for the small-size behaviour of the cluster size distribution are shown in figures $5(d-f)$. The small- $x$ behaviour appears to obey scaling, although the quality of the agreement between theory and simulations is not as good as in the large-size limit. Generally, for a larger value of $\lambda$, there is a faster approach to the asymptotic limit, and better agreement between theory and simulations.

\section{Discussion}

A general treatment of linear and collision-induced fragmentation processes has been given within the framework of the rate equations. This is an approach of a mean-field character, as fluctuations in the spatial positions of the clusters and in cluster shape are ignored. The microscopic details of breakup events are accounted for by the overall breakup rate, which that specifies the likelihood for a fragment of a given mass to break (either by an external agent or by collisions), and by the relative breakup rate, which gives the size distribution of products from a single breakup event.

For a linear, homogeneous fragmenting system scaling solutions exist when the homogeneity index, $\lambda$, of the overall breakup rate is positive. Within the scaling regime, the value of $\dot{\lambda}$ is the crucial parameter which characterises the large-mass behaviour of the cluster size distribution. In the small-mass limit, the cluster size distribution is determined by whether or not the relative breakup rate is cut off in the small-size limit. With a cutoff, the cluster size distribution is sharply peaked, while in the opposite case, there is a considerable population of very small dust-like particles. When $\lambda<0$, a shattering transition occurs in which mass is lost to a shattered phase consisting of zero-mass particles. For this case, it is possible to write a scaling ansatz for the cluster size distribution, but one in which the initial mass plays the role of a characteristic mass scale. Owing to the rapid breakup of very small clusters, the initial mass decays away extremely slowly, so that this component of the distribution acts as a steady source.

To gain some insight into the behaviour that can occur when nonlinear effects play a role in particle breakup, a class of collision-induced 'splitting' models was introduced, in which a fragmenting cluster always breaks into two equal-sized pieces. These models exhibit the essential nonlinear nature of collision-induced breakup, but are simple enough to analyse. In model I, both colliding fragments split in two upon collision, while in models II and III, only the larger, or only the smaller, respectively, of the two colliding fragment splits in two. For these models, scaling is valid for different ranges of $\lambda$. Model III is unique in that large clusters are 'frozen out' of the fragmentation process 

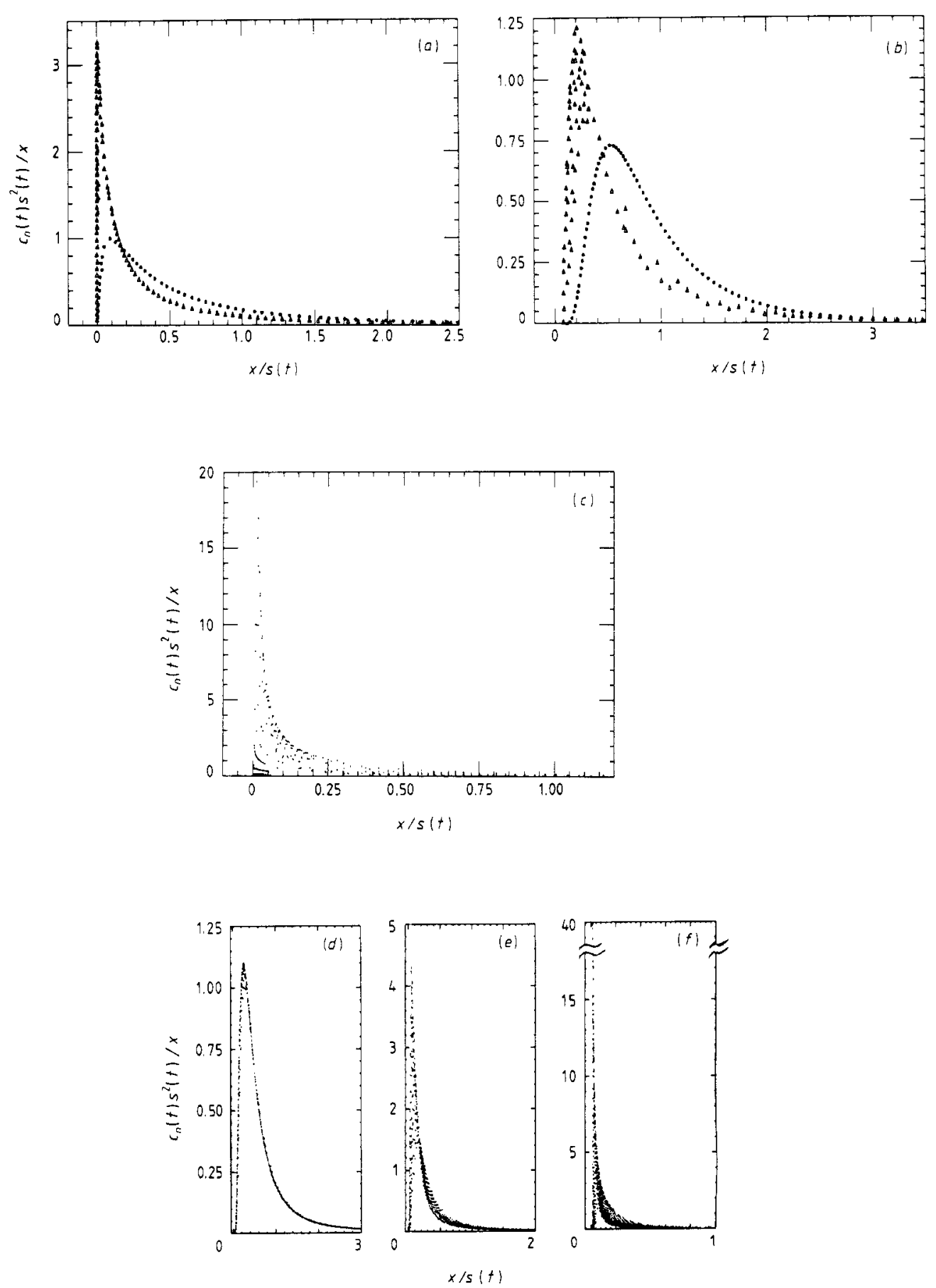

Figure 4. Scaling plot of $c_{n}(t)$. Shown is the scaled quantity $\left(s(t)^{2} / x\right) c_{n}(t)$ against $x / s(t)$, where $x=2^{-n}$, for various values of $\lambda$. In each plot $s(t)$ ranges from approximately $10^{-1}$ to $10^{-8}$. We show data for: (a) model I for $\lambda=0.5(*)$, and $0.25(\Delta),(b)$ model II for $\lambda=0.5$ (*) and $0.0(\Delta),(c)$ model II for $\lambda=-0.25$, and $(d),(e),(f)$ model III for $\lambda=1.5,1.0,0.75$ respectively. Data collapsing begins to fail for the smaller values of $\lambda$, from which one can infer the end of the scaling regime. 

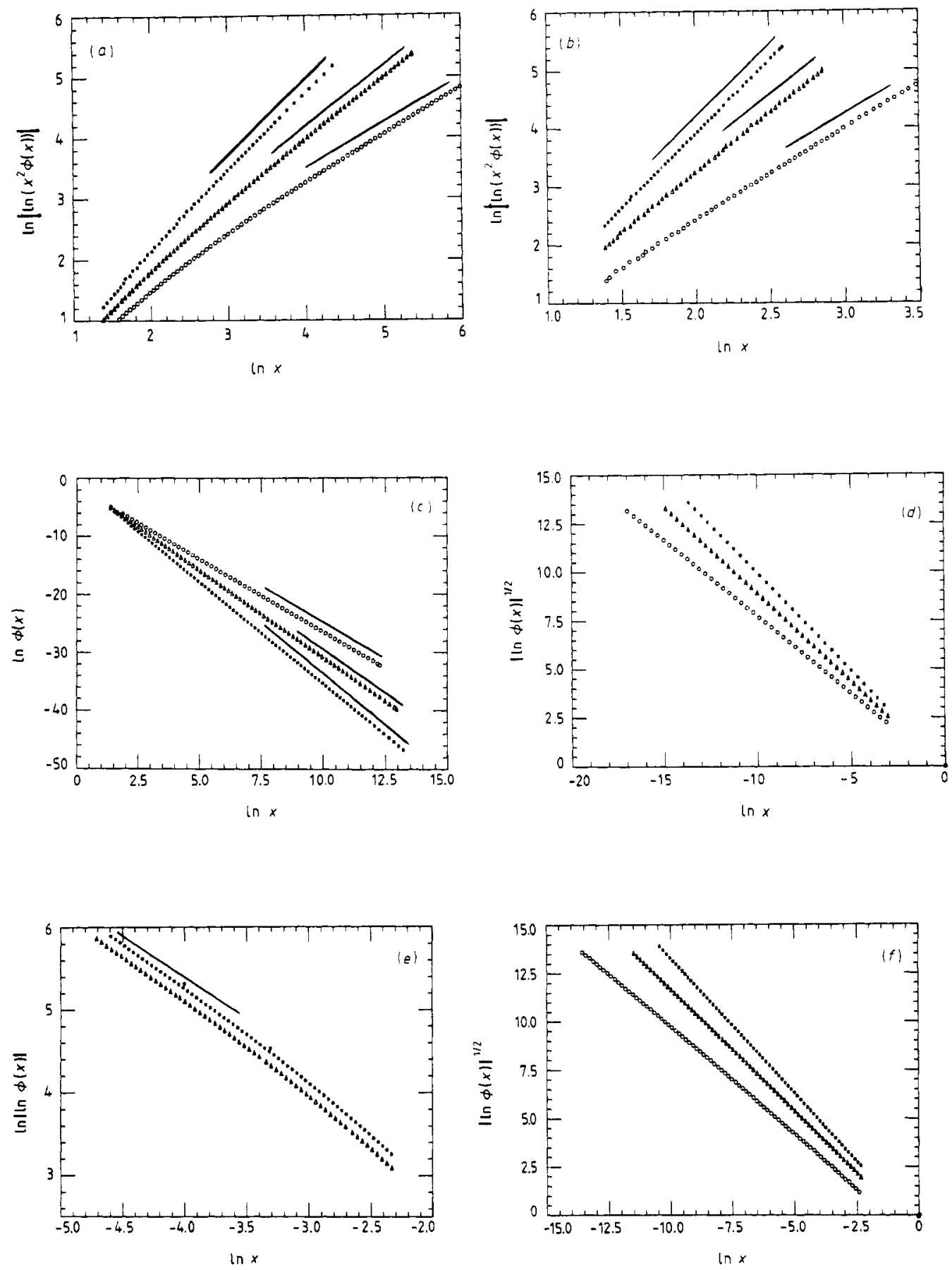

Figure 5. Asymptotic behaviours of $\phi(x)$ in both $(a)-(c)$ the large-mass limits for models I-III respectively, and $(d)-(f)$ in the small-mass limits for models I-III respectively. Data for the cases $\lambda=1.5(0), 2.0(\Delta)$ and $2.5(*)$ are shown. The full lines in $(a)-(c)$ indicate the slopes predicted by equations $(47 a),(55 b)$ and $(69 a)$ for models I-III respectively. In $(d)$ and $(f)$, the data are consistent with a log-normal tail. In (e), the line has slope -1 (equation 55(b)), and the data for the case of $\lambda=1.5$ are not shown. 
at long time. This leads to a monotonic fragment size distribution, with a power-law tail at large masses, while the distributions in models I and II are non-monotonic and decay quasi-exponentially for large mass. Moreover, in model III the concentration of fragments of any finite size decays as a power law in time, and in models I and II the concentrations decay exponentially.

While the rate equation approach provides a useful and comprehensive account of a wide range of fragmentation phenomena, there are interesting questions of potential experimental relevance that are worth addressing. It is important to develop approximations which can take inhomogeneities of various types into account. While the assumption of perfect mixing is probably appropriate for ball-milling types of comminution processes [7], it is clearly inadequate in many geophysical situations, for example, where fragments remain in fixed spatial positions throughout the breakup process. Attempts to account for this type of spatial inhomogeneity have been attempted, but primarily at a qualitative level [27]. It is also clear that the rate of fragmentation processes should generally depend on the cluster shape. Whether this shape dependence can be accounted for by averaging over fragment shapes is not understood. Finally, in many fragmentation processes there are various nonlinear and non-local effects. When breakup is driven by high pressures, there can be a transfer of stress across many fragments. When fragments possess considerable kinetic energy, nonlinear effects, induced by collisions may play a substantial role. The understanding of the kinetics in these types of systems should pose rich areas for new research.

\section{Acknowledgments}

We wish to thank E Guyon for useful discussions and R Ziff for helpful correspondence and for sending us Filippov's paper. This work has been supported in part by grant no DAAL03-89-K-0025 from the Army Research Office. We gratefully acknowledge this financial support.

\section{References}

[1] Montroll E W and Simha R 1940 J. Chem. Phys. 8721

[2] Blatz P J and Tobolsky A V 1945 J. Phys. C: Solid State Phys. 4977

[3] Jellinek $\mathrm{H} \mathrm{H}$ and White G $1951 \mathrm{~J}$. Polymer. Sci. 6745 Saito O 1958 J. Phys. Soc. Japan 13198

[4] Basedow A M, Ebert K H and Ederer H J 1978 Macromolecules 11774

[5] Shinnar R $1961 J$. Fluid Mech. 10259

[6] Gilvarry J J 1961 J. Appl. Phys. 32391

[7] Austin L, Shoji K, Bhatio V, Savage K and Klimpel R 1976 Ind. Eng. Chem. Process Des. Dev. 15187

[8] Sahimi M and Tsotsis T T 1987 Phys. Rev. Lett. 59888

[9] Filippov A F 1961 Theory Probab. Appl. 4275

[10] Ziff R M and McGrady E D 1985 J. Phys. A: Math. Gen. 183027

[11] Ziff R M and McGrady E D 1986 Macromolecules 192513

[12] McGrady E D and Ziff R M 1987 Phys. Rev. Lett. 58892

[13] Peterson T W, Scotto M V and Sarofim A F 1985 Powder Tech. 4587

[14] Peterson T W 1986 Aerosol Sci. Tech. 593

[15] Kerstein A R 1986 Dynamic scaling for comminution preprint Sandia National Laboratories

[16] Edwards B F, Cai M and Han H 1990 Phys. Rev. A in press Cai M, Edwards B F and Han H 1990 Phys. Rev. A in press

[17] Cheng $Z$ and Redner S 1988 Phys. Rev. Lett. 602450 
[18] Friedlander S K and Wang C S 1966 J. Colloid Interface Sci. 22126

[19] Vicsek T and Family F 1984 Phys. Rev. Lett. 521669

[20] van Dongen P G D and Ernst M H 1985 Phys. Rev. Lett. 541396

[21] Kang K, Redner S, Meakin P and Leyvraz F 1986 Phys. Rev. A 331171

[22] Siebesma A P, Tremblay R R, Erzan A and Pietronero L 1989 Physica 156A 613

[23] Srivastava R C 1982 J. Atmos. Sci. 391317

[24] Kolmogorov A N 1941 Dokl. Akad. Nauk. 3199

[25] Aitcheson J 1957 The Log-Normal Distribution (Cambridge: Cambridge University Press)

[26] Montroll E W and Shlesinger M F 1984 Nonequilibrium Phenomena II: From Stochastics to Hydrodynamics ed J L Lebowitz and E W Montroll (Amsterdam: North-Holland)

[27] Turcotte D L 1986 J. Geophys. Res. 911921 